\title{
Síntesis de Espumas Rígidas de Poliuretano Obtenidas a partir de Aceite de Castor y Poliglicerol Empleando Calentamiento por Microondas
}

\author{
Paula Mazo, Omar Yarce, Luis A. Rios \\ Departamento de Ingeniería Química, Universidad de Antioquia
}

\begin{abstract}
Resumen: En este trabajo se realizó la síntesis de espumas rígidas de poliuretano utilizando un macropoliol sintetizado a partir de aceite de castor y poliglicerol, se optimizó mediante un análisis de superficie de respuesta, empleando un diseño factorial $3^{2}$, los factores evaluados fueron: cantidad de agua y de ácido tartárico, el cual actúa como agente entrecruzante y catalizador; se evalúaron las propiedades mecánicas de las espumas como: densidad aparente (ASTM D1622-08), resistencia a la compresión (ASTM D1621), conductividad térmica (ASTM C177-04) y friabilidad (ASTM C421-8); se realizó Microscopia Electrónica de Barrido (SEM) y Análisis de Calorimetría Diferencial de Barrido (DSC). El macropoliol fue obtenido desde fuentes renovables, mediante la transesterificación de Aceite de Castor (CO) ó Aceite de Castor Maleinizado (MACO) con Poliglicerol (PG). Se evalúo el efecto de las microondas en las reacciones, donde se encuentra una disminución del tiempo; el seguimiento y la caracterización de los productos intermedios se realiza mediante la cuantificación de valor ácido por titulación (ASTM D4662-03) y número de hidroxilos (ASTM D4274-05).
\end{abstract}

Palabras claves: Poliuretano, espuma rígida, aceite castor, poliglicerol.

\section{Synthesis of Rigid Polyurethane Foams Obtained from Castor Oil and Polyglycerol Using Microwave Heating}

\begin{abstract}
In this work we showed the polyurethane rigid foams synthesis using a macropolyol synthesized from castor oil and polyglycerol. This process is optimized employing a response surface analysis and using a $3^{2}$ factorial design. The factors evaluated were: amount of water and tartaric acid, where tartaric acid acts as crosslinking agent and catalyst; mechanical properties of foams were evaluated as bulk density (ASTM D1622-08), compressive strength (ASTM D1621), thermal conductivity (ASTM C177-04), and friability (ASTM C421-8); scanning electronic microscopic (SEM) and differential scanning calorimetry (DSC). The macropolyol was obtained from renewable sources by the transesterification of castor oil (CO) or maleinized castor oil (MACO) and polyglycerol (PG). The effect of microwaves on the reactions was evaluated, where there was a decrease in time. The monitoring and characterization of intermediate products were performed by quantifying the acid value by titration (ASTM D4662-03) and hydroxyl number (ASTM D4274-05).
\end{abstract}

Keywords: Polyurethane, rigid foam, castor oil, polyglycerol.

\section{Introducción}

Debido al constante desarrollo industrial, se ha generado un creciente interés por fuentes alternas que provean materias primas técnicamente viables y ambientalmente sostenibles para ser empleadas en los diferentes procesos tecnológicos ${ }^{[1,2]}$.

Una alternativa importante podría ser la generación de nuevos materiales y compuestos poliméricos, tal como el poliuretano que es muy versátil, de excelentes propiedades mecánicas y químicas, y se puede emplear en su síntesis monómeros derivados de fuentes renovables ${ }^{[3]}$

Los poliuretanos (PU) fueron descubiertos por Otto Bayer en 1937, pero solo a partir de 1946 después de la segunda guerra mundial se presentó un crecimiento enorme de estos compuestos debido a la gran demanda. Se han desarrollado un gran número de aplicaciones en diversos segmentos de mercado, por ejemplo, las espumas flexibles se usan en la producción de colchones, tapizados y asientos para la industria automotriz; las espumas rígidas se usan principalmente como aislantes térmicos en equipos de refrigeración, camiones frigoríficos y como material estructural para la industria de la construcción. Además se tienen los poliuretanos sólidos usados como elastómeros, tintas y/o revestimientos, adhesivos y/o pegantes, fibras, sellantes, impermeabilizantes, etc ${ }^{[4,5]}$.

Las espumas rígidas de PU, representan el segundo mayor mercado de los PU a nivel mundial, después de las espumas flexibles ${ }^{[5]}$. Son formuladas generalmente a partir de dos componentes. Uno de ellos es el isocianato (normalmente el MDI Polimérico) y el otro un macropoliol, además de los aditivos como: surfactantes, retardantes de llama, catalizadores y agentes de expansión como compuestos halocarbonados ${ }^{[6]}$.

El aceite de castor (CO) y aceite de castor maleinizado (MACO) se ha empleado como poliol en trabajos anteriores para obtener espumas flexibles de $\mathrm{PU}^{[7,8]}$ y adhesivos ${ }^{[9]}$, pero estos no tienen la suficiente cantidad de hidroxilos para aplicaciones en espumas rígidas, por lo cual se propone transesterificarlos con poliglicerol empleando microondas para obtener un producto biodegradable, de propiedades equivalentes a las espumas petroquímicas y sin emplear agentes de expansión halocarbonados.

El poliglicerol es un poliol polieter que puede ser producido por diversos métodos, algunos de ellos son: reacción de glicerol con epiclorhidrina ${ }^{[10]}$, glicerol con glicidol $^{[1,12]}$ y por autocondensación del glicerol empleando catalizadores ácidos ó básicos con eliminación de agua ${ }^{[13]}$. El poliglicerol es una mezcla compleja de oligómeros lineales, ramificados y cíclicos, donde la distribución varía de acuerdo al método de producción ${ }^{[14]}$. Es insoluble en solventes orgánicos como éter, cetonas, aromáticos, compuestos halogenados; solamente es soluble en compuestos con alta polaridad como agua, alcoholes, solventes apróticos dipolares (DMF ó DMSO).

Autor para correspondência: Paula Mazo, Grupo Procesos Fisicoquímicos Aplicados, Departamento de Ingeniería Química, Universidad de Antioquia, Calle 67, № 53-108, Medellín, Colômbia. E-mail: pcmazo@matematicas.udea.edu.co 
Debido al alto costo y limitada compatibilidad con muchos compuestos orgánicos el poliglicerol no ha sido empleado en la industria de los poliuretanos. Más recientemente debido a la alta producción de glicerol debido a la industria del biodiesel, se ha generado gran interés en generar polioles a partir de este ${ }^{[15]}$.

Zoran et al. (2009), ha producido polioles basados en poliglicerol para la producción de poliuretanos, donde utiliza policondensación de glicerol en presencia de catalizadores alcalinos a temperaturas de 240-270 ${ }^{\circ} \mathrm{C}$, entre 4-6 horas y luego se realiza la transesterificación con aceites vegetales a $160-250{ }^{\circ} \mathrm{C}$, en el mismo tiempo, el poliol obtenido se utiliza para la producción de espumas rígidas de baja densidad $^{[16]}$.

Las microondas son radiación electromagnética no ionizante, que no causa cambios en la estructura molecular pero producen movimiento molecular, por migración de iones y rotación de dipolos en materiales dieléctricos, que generan fricción por las colisiones moleculares lo que hace que el material se caliente. Tiene un rango de frecuencia desde $300 \mathrm{MHz}$ a $300 \mathrm{GHz}$ y la correspondiente longitud de onda de $1 \mathrm{~m}$ a $1 \mathrm{~mm}$. La frecuencia más usada a nivel industrial y doméstico es la $2.45 \mathrm{GHz}^{[17,18]}$.

Los sistemas polares que siguen la ecuación de Arrhenius, expresada por la Ecuación 1, pueden ser afectados cuando son sometidos a irradiación con microondas, porque aumenta el factor pre-exponencial A y se disminuye el cambio en la energía libre de Gibbs del estado de transición $\left(\Delta \mathrm{G}^{\neq}\right)$debido al aumento de la probabilidad de impactos moleculares por aumento de la polarización dipolar de las moléculas con el campo eléctrico.

$$
k=A^{*} e^{\left(-\Delta G^{\neq} / R T\right)}
$$

Mazo et al. ${ }^{[19-21]}$ han estudiado el efecto que tienen las microondas en las reacciones de esterificación y transesterificación de aceites vegetales, las cuales se ven favorecidas, disminuyendo el tiempo y las condiciones de reacción.

En este trabajo se propone estudiar la cinética de la reacción de maleinización del aceite de castor, la policondensación del glicerol y la transesterificación del poliglicerol obtenido con aceite de castor y MACO, empleando microondas, para determinar efectos específicos no térmicos en estas reacciones. También con el poliol obtenido sintetizar espumas rígidas de poliuretano y caracterizarlas mediante pruebas mecánicas y químicas.

\section{Experimental}

\section{Reactivos y métodos}

Para las reacciones se emplearon los siguientes reactivos: aceite de castor (CO) grado USB, con un valor de $\mathrm{OHv}=159.51 \mathrm{mgKOH} / \mathrm{g}$ muestra, anhidrido maleíco grado comercial (Merk), glicerol grado comercial (Merk), $\mathrm{NaOH}(\mathrm{s})$ (Merk), ácido tartárico (Merk), difenil metano diisocianato (MDI) grado comercial (Huntsman), silicona con un porcentaje de siloxano de $25-35 \%$ (General Electric) y un peso molecular de 6000, agua corriente sin ningún tratamiento, trietil amina (TEA) grado comercial (Tego), octoato de estaño grado comercial (Tego), tolueno grado analítico (Merk).

\section{Diseño experimental}

\section{Sistemas de reacción y calentamiento convencional y microondas}

La Figura 1 muestra el esquema de reacción empleando calentamiento convencional con una manta y el calentamiento con microondas utilizando un horno Samsung modificado. En los dos sistemas el control y medición de temperatura se realiza con un controlador Maxthermo MC5438 y una termocupla tipo J, con variación de $+/-1{ }^{\circ} \mathrm{C}$. El sistema de control se realiza mediante encendido y apagado cuando la temperatura se encuentra en el valor programado, para evitar la dependencia de la potencia esta se mantuvo en un valor constante de $100 \%$, el horno tiene una potencia de salida de 800 W. La agitación es mecánica empleando un motor con variación de velocidad.

\section{Síntesis y caracterización del aceite de castor modificado}

El anhídrido maléico y el aceite de castor fueron adicionados en una relación molar 1:1 en un reactor de $250 \mathrm{~mL}$ de vidrio con alimentación constante de nitrógeno y agitación mecánica. Se varió el tiempo, la temperatura de reacción fue de $100{ }^{\circ} \mathrm{C}$. Se tomaron muestras a lo largo de la reacción, se lavaron con agua destilada y secaron con sulfato de magnesio anhidro, para determinar el valor ácido por titulación (ASTM D4662-03).

\section{Síntesis y caracterización de poliglicerol}

Se cargan $300 \mathrm{~g}$ de glicerol con $3 \mathrm{~g}$ de $\mathrm{NaOH}$ (1\% en peso), en un reactor de vidrio con entrada de nitrógeno, agitador mecánico y vacio para extraer la mezcla agua + glicerina formada

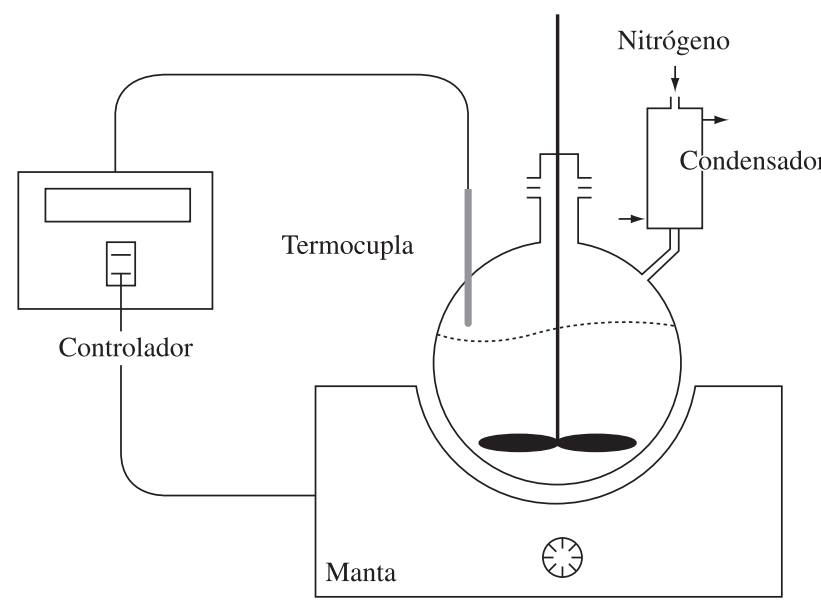

Convencional

(a)

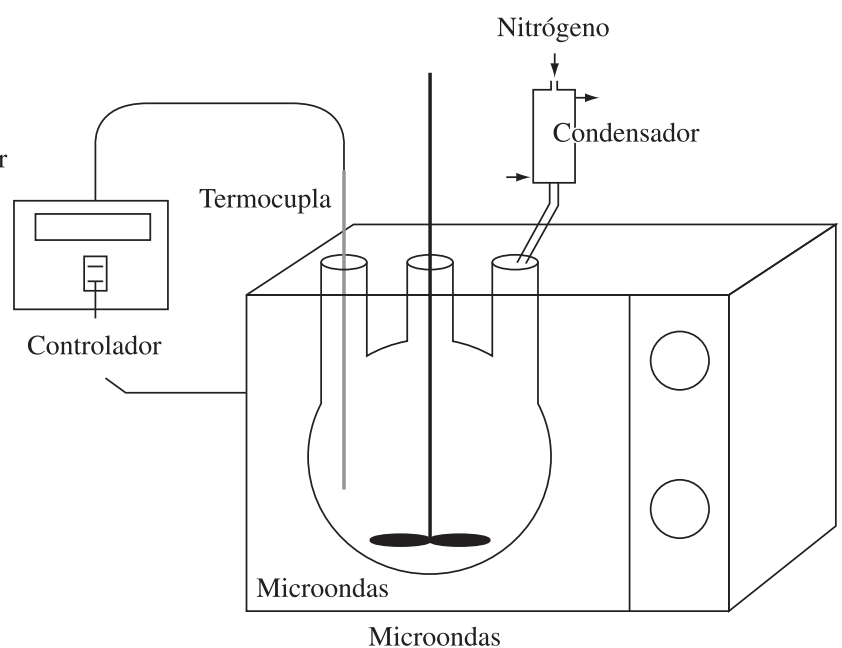

(b)

Figura 1. Representación esquemática del reactor $(250 \mathrm{~mL})$ usando calentamiento convencional (a) y microondas (b). 
por la autocondensación. Se evaluó una temperatura de $200{ }^{\circ} \mathrm{C}$, empleando el horno microondas y la manta de calentamiento descritas anteriormente. El seguimiento de la reacción se realiza midiendo el número de hidroxilos (ASTM D4274-05), las muestras son neutralizadas con $\mathrm{HCl} 1 \mathrm{~N}$, antes de los análisis.

\section{Síntesis y caracterización de los aceites transesterificados con poliglicerol}

Se cargan $120 \mathrm{~g}$ de CO ó MACO con $80 \mathrm{~g}$ de poliglicerol sin neutralizar, en un reactor de vidrio con entrada de nitrógeno y agitador mecánico. Se evaluó una temperatura de $160{ }^{\circ} \mathrm{C}$, empleando el horno microondas y la manta de calentamiento descritas anteriormente. Se evalúa el tiempo necesario para que se forme una sola fase en el sistema. Se cuantifica el número de hidroxilos (ASTM D4274-05).

\section{Síntesis y caracterización de las espumas rígidas}

Para la síntesis de las espumas rígidas se realizó un diseño factorial $3^{2}$ con cuatro repeticiones al centro y se optimiza mediante

Tabla 1. Variables y niveles empleados en la preparación de las espumas rígidas.

\begin{tabular}{ccccc}
\hline & Variables & \multicolumn{3}{c}{ Niveles } \\
\cline { 3 - 5 } & & $\mathbf{- 1}$ & $\mathbf{0}$ & $\mathbf{1}$ \\
\hline 1 & Agua & 1 & 2 & 3 \\
2 & \% Tartárico* & 0.00 & 1.75 & 3.50 \\
\hline
\end{tabular}

Agua: cantidad de agua en partes por 100 poliol (pph); \% TARTÁRICO: porcentaje en peso respecto a la cantidad de poliol que se empleó en cada ensayo. la metodología de superficie de respuesta. Los variables y niveles se especifican en la Tabla 1, el diseño experimental en la Tabla 2.

El análisis estadístico se realiza empleando el software StatGraphics 5.1, empleando una ecuación polinomial de segundo orden:

$$
z=\beta_{0}+\beta_{1} x+\beta_{2} y+\beta_{3} x^{2}+\beta_{4} y^{2}+\beta_{5} x y
$$

donde:

- z: variable de respuesta: densidad o resistencia a la compresión;

- $\mathrm{x}$ : variable independiente codificada de la cantidad de agua en partes por 100 poliol (pph);

- y: variable codificada correspondiente al porcentaje en peso de ácido tartárico respecto a la cantidad de poliol que se empleó en cada ensayo (\%tartárico);

- $\beta_{0}, \beta_{1}, \beta_{2}, \beta_{3}, \beta_{4}, \beta_{5}$ son los coeficientes respectivos del intercepto, lineal, cuadrática e interacción.

Para determinar el punto estacionario se calcula la derivada de la superficie de respuesta con respecto a cada una de las variables codificadas y se resuelve el sistema de ecuaciones, luego se calculan los valores reales.

$$
\frac{\partial Z}{\partial y}=0 \Rightarrow y \quad \frac{\partial Z}{\partial x}=0 \Rightarrow x
$$

Para determinar si el punto estacionario corresponde a un máximo, mínimo o punto silla, se debe emplear la matriz Hessiana, la cual es:

\begin{tabular}{|c|c|c|c|c|c|c|c|}
\hline & \multicolumn{7}{|c|}{ Diseño de experimentos } \\
\hline & Ensayo & VBL 1 & VBL 2 & POLIOL $(\mathrm{g})$ & Agua $(g)$ & Tartárico (g) & MDI (g) \\
\hline \multirow{14}{*}{$\begin{array}{l}0 \\
\bigcup \\
0 \\
0\end{array}$} & 1 & -1 & -1 & 100 & 1 & 0 & 128,39 \\
\hline & 2 & 1 & -1 & 100 & 3 & 0 & 161,49 \\
\hline & 3 & 0 & 1 & 100 & 2 & 3.5 & 151,9 \\
\hline & 4 & 1 & 0 & 100 & 3 & 1.75 & 164,97 \\
\hline & 5 & 0 & -1 & 100 & 2 & 0 & 144,94 \\
\hline & 6 & 1 & 1 & 100 & 3 & 3.5 & 168,45 \\
\hline & 7 & 0 & 0 & 100 & 2 & 1.75 & 148,42 \\
\hline & 8 & -1 & 1 & 100 & 1 & 3.5 & 135,35 \\
\hline & 9 & -1 & 0 & 100 & 1 & 1.75 & 131,87 \\
\hline & 10 & 0 & 0 & 100 & 2 & 1.75 & 148,42 \\
\hline & 11 & 0 & 0 & 100 & 2 & 1.75 & 148,42 \\
\hline & 12 & 0 & 0 & 100 & 2 & 1.75 & 148,42 \\
\hline & 13 & 0 & 0 & 100 & 2 & 1.75 & 148,42 \\
\hline & 14 & 0 & 0 & 100 & 2 & 1.75 & 148,42 \\
\hline \multirow{14}{*}{ } & 15 & -1 & -1 & 100 & 1 & 0 & 116,17 \\
\hline & 16 & 1 & -1 & 100 & 3 & 0 & 149,27 \\
\hline & 17 & 0 & 1 & 100 & 2 & 3.5 & 135,48 \\
\hline & 18 & 1 & 0 & 100 & 3 & 1.75 & 152,75 \\
\hline & 19 & 0 & -1 & 100 & 2 & 0 & 132,72 \\
\hline & 20 & 1 & 1 & 100 & 3 & 3.5 & 156,23 \\
\hline & 21 & 0 & 0 & 100 & 2 & 1.75 & 136,20 \\
\hline & 22 & -1 & 1 & 100 & 1 & 3.5 & 123,13 \\
\hline & 23 & -1 & 0 & 100 & 1 & 1.75 & 119,65 \\
\hline & 24 & 0 & 0 & 100 & 2 & 1.75 & 136,20 \\
\hline & 25 & 0 & 0 & 100 & 2 & 1.75 & 136,20 \\
\hline & 26 & 0 & 0 & 100 & 2 & 1.75 & 136,20 \\
\hline & 27 & 0 & 0 & 100 & 2 & 1.75 & 136,20 \\
\hline & 28 & 0 & 0 & 100 & 2 & 1.75 & 136,20 \\
\hline
\end{tabular}

Tabla 2. Cantidades de reactivos para la obtención de las espumas rígidas. 


$$
H=\left|\begin{array}{ll}
\frac{\partial^{2} Z}{\partial^{2} x} & \frac{\partial^{2} z}{\partial x \partial y} \\
\frac{\partial^{2} z}{\partial y \partial x} & \frac{\partial^{2} z}{\partial^{2} y}
\end{array}\right|
$$

Y se calculan los determinantes de las submatrices (Det), dependiendo del signo obtenido podemos clasificar el punto estacionario así:

- Det $\mathrm{H}_{1}<0$ y Det $\mathrm{H}_{2}>0$, el punto es un máximo;

- Det $\mathrm{H}_{1}>0$ y Det $\mathrm{H}_{2}>0$, el punto es mínimo;

- En otros casos es silla.

La relación molar de grupos isocianato a grupos hidroxilo (NCO:OH) permanecerá constante en un valor de 1.1, la cantidad de catalizadores SO y TEA fue de $0.2 \mathrm{~g}$, la silicona fue de $1 \mathrm{~g}$.

Las variables respuesta para estos experimentos fueron: densidad aparente (ASTM D1622-08) y resistencia a la compresión (ASTM D1621), para las espumas óptimas se evaluaron otras propiedades como: conductividad térmica (ASTM C177-04) y friabilidad (ASTM C421-8).

Los espectros IR se realizaron en un espectrofotómetro marca Perkin Elmer y la morfología de la superficie de las espumas fue observada en un microscopio de escaneo de electrones (scanning electron microscope. SEM) Hitachi S-520. Las espumas fueron cortadas en capas de $2 \mathrm{~mm}$ de espesor y recubiertas con una capa de oro. Se realizó análisis de calorimetría diferencial de barrido (DSC) empleando un equipo TA Instrument a una velocidad de calentamiento de $10{ }^{\circ} \mathrm{C} / \mathrm{min}$.

\section{Resultados y Discusión}

\section{Influencia de las MW en la obtención de MACO}

Como puede observarse en la Figura 2, la reacción de maleinización del aceite de Castor tiene un comportamiento y valores similares de acidez, empleando calentamiento convencional (CONV) y microondas (MW), por lo cual no existen efectos no térmicos que afecten el mecanismo de la reacción, esto puede ser debido a la baja polaridad de los reactivos.

\section{Influencia de las MW en la obtención de Poliglicerol}

En la Figura 3 se puede observar que las MW ejercen un efecto directo en la disminución del valor de hidroxilos, solo se requiere de 2 horas para obtener un poliglicerol con $850.7 \mathrm{mg} \mathrm{KOH} / \mathrm{g}$, que corresponde con un decaglicerol, mientras que con calentamiento convencional en 6 horas de reacción solo ha disminuido hasta $924.8 \mathrm{mg} \mathrm{KOH} / \mathrm{g}$, que corresponde con un hexaglicerol, estas son aproximaciones basadas en estudios anteriores ${ }^{[14]}$.

Esto puede ser debido a la alta polaridad de los reactivos y del mecanismo de la reacción, los intermedios son oxianiones y carbocationes, que tienen una alta constante dieléctrica e interactúan con el campo eléctrico, aumentando la polarización dipolar y la agitación molecular, que conlleva a un incremento acelerado de la temperatura localizada. Debe existir una excelente agitación para minimizar los puntos calientes que se generan, por esta razón se emplea agitación mecánica.

Los espectros IR obtenidos para la glicerina pura y el poliglicerol (Figura 4), muestran que las señales características en ambos se dan en: $3398 \mathrm{~cm}^{-1}$ estiramiento $\mathrm{OH}, 2939$ y $2890 \mathrm{~cm}^{-1}$ estiramientos - $\mathrm{CH}$ - alcano, 1458 y $1418 \mathrm{~cm}^{-1}$ flexiones simétricas de los grupos $\mathrm{CH}_{2}, 1242,1112$ y $1051 \mathrm{~cm}^{-1}$ corresponden con estiramientos de -C-O-. En el poliglicerol aparece una señal adicional a $1642 \mathrm{~cm}^{-1}$ de grupos $-\mathrm{C}=\mathrm{C}-$, de dobles enlaces formados por reacciones de eliminación de agua intermolecular, debido a la alta temperatura de

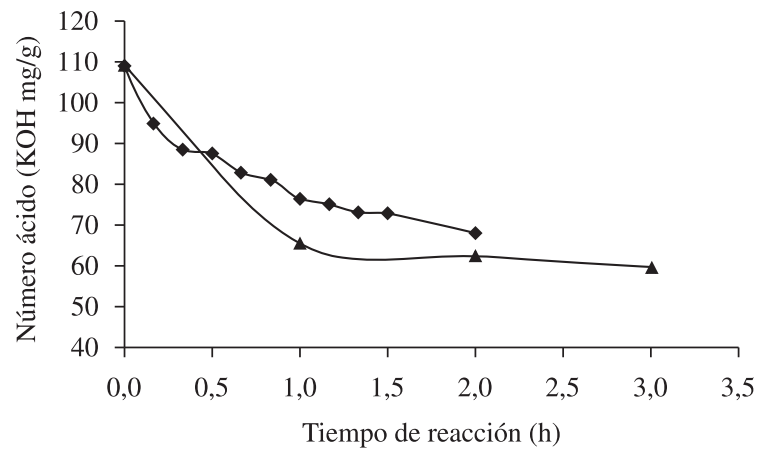

$\neg$ Maco conv $100 \mathrm{C} \longrightarrow$ Maco MW $100 \mathrm{C}$

Figura 2. Seguimiento del valor de acidez en la reacción de maleinización de CO con anhídrido maleico, relación molar (1:1), temperatura $100{ }^{\circ} \mathrm{C}$.

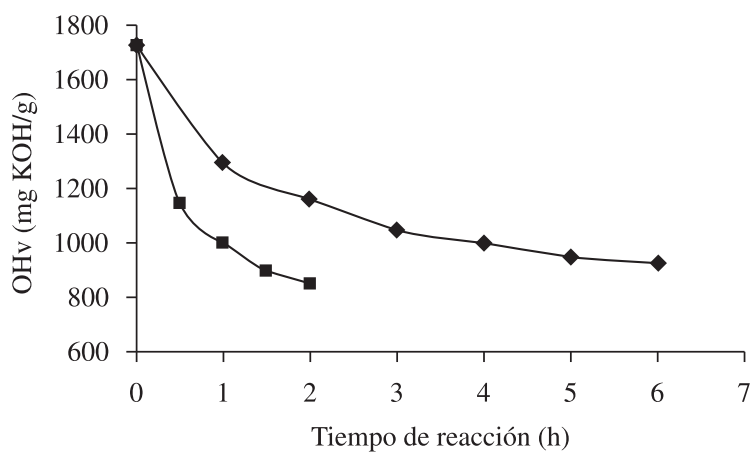

$\mathrm{PG}$ CONV $200 \mathrm{C} \rightarrow \mathrm{PG}$ MW $200 \mathrm{C}$

Figura 3. Seguimiento del valor de hidroxilos (OHv) en la reacción de policondensación de glicerol con $\mathrm{NaOH}$, temperatura $200{ }^{\circ} \mathrm{C}$.

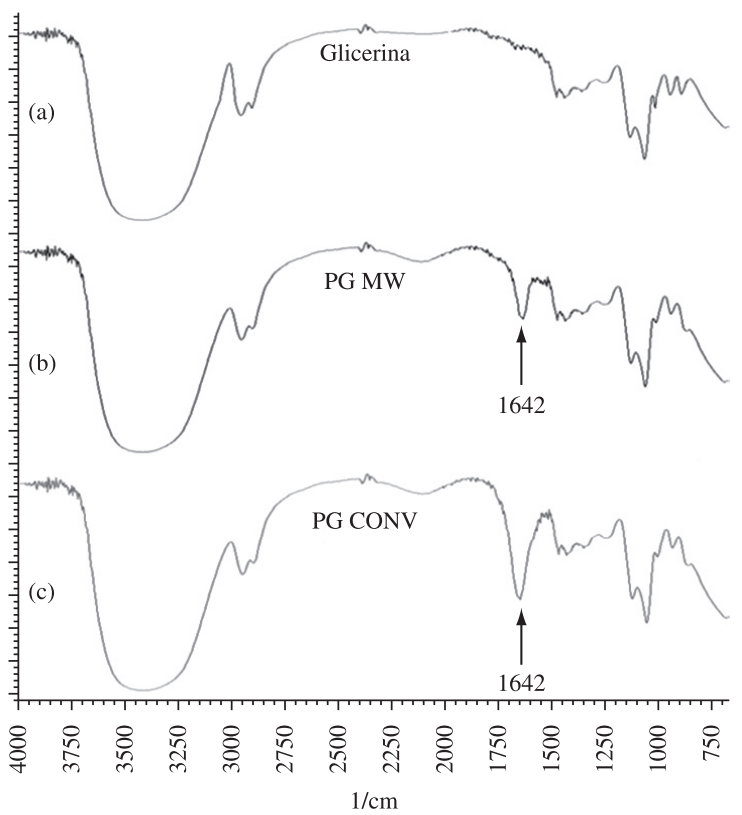

Figura 4. Espectros IR de glicerina (a), poliglicerol con MW (b), poliglicerol con CONV (c).

reacción, en el caso de emplear calentamiento convencional, este pico tiene mayor intensidad, ya que existe un tiempo de reacción más prolongado. 
Tabla 3. Caracterización de los aceites obtenidos mediante transesterificación de CO ó MACO con PG, temperatura $160^{\circ} \mathrm{C}$.

\begin{tabular}{cccccc}
\hline Poliol & $\begin{array}{c}\text { Método de } \\
\text { calentamiento }\end{array}$ & $\begin{array}{c}\text { Tiempo }(\mathbf{h}) \text { para } \\
\text { obtener } \mathbf{~ f a s e}\end{array}$ & $\begin{array}{c}\text { OHv } \\
(\mathbf{m g ~ K O H} / \mathbf{g}) \text { teórico }\end{array}$ & $\begin{array}{c}\text { OHv } \\
\text { (mg KOH/g) real }\end{array}$ & $\begin{array}{c}\text { Viscosidad } \\
\text { (cps) }\end{array}$ \\
\hline PG-CO & CONV & 6 & 436.27 & 434.21 & 4200 \\
PG-CO & MW & 2 & 436.27 & 421.67 & 4300 \\
PG-MACO & CONV & 6 & 400.268 & 390.64 & 4500 \\
PG-MACO & MW & 2 & 400.268 & 375.56 & 4500 \\
\hline
\end{tabular}

Tabla 4. Valores de las propiedades evaluadas en las espumas.

\begin{tabular}{cccccc}
\hline & \multicolumn{2}{c}{ PG-CO } & \multicolumn{2}{c}{ PG-MACO } \\
\hline $\mathbf{x}$ & $\mathbf{y}$ & $\begin{array}{c}\text { Densidad } \\
\text { Kg.m }^{-3} \\
\text { D }\end{array}$ & $\begin{array}{c}\text { Resistencia } \\
\text { compresión } \\
\text { KPa } \\
\text { RC }\end{array}$ & $\begin{array}{c}\text { Densidad } \\
\text { Kg.m }^{-3} \\
\text { D }\end{array}$ & $\begin{array}{c}\text { Resistencia } \\
\text { Compresión } \\
\text { KPa } \\
\text { RC }\end{array}$ \\
\hline-1 & -1 & 82.3 & 165.4 & 79.7 & 171.6 \\
1 & -1 & 45.8 & 137.2 & 40.9 & 143.4 \\
0 & 1 & 71.4 & 257.4 & 70.0 & 261.4 \\
1 & 0 & 51.3 & 192.5 & 50.4 & 200.5 \\
0 & -1 & 65.8 & 154.3 & 64.6 & 162.3 \\
1 & 1 & 57.2 & 223.7 & 55.5 & 240.4 \\
0 & 0 & 67.2 & 231.4 & 65.3 & 240.2 \\
-1 & 1 & 94.6 & 283.4 & 93.1 & 292.1 \\
-1 & 0 & 91.2 & 233.4 & 89.2 & 242.3 \\
0 & 0 & 70.4 & 241.8 & 65.3 & 250.8 \\
0 & 0 & 72.3 & 253.2 & 71.7 & 263.1 \\
0 & 0 & 69.4 & 261.4 & 69.8 & 249.8 \\
0 & 0 & 63.7 & 239.7 & 61.9 & 251.4 \\
0 & 0 & 80.2 & 245.2 & 72.1 & 261.4 \\
\hline
\end{tabular}

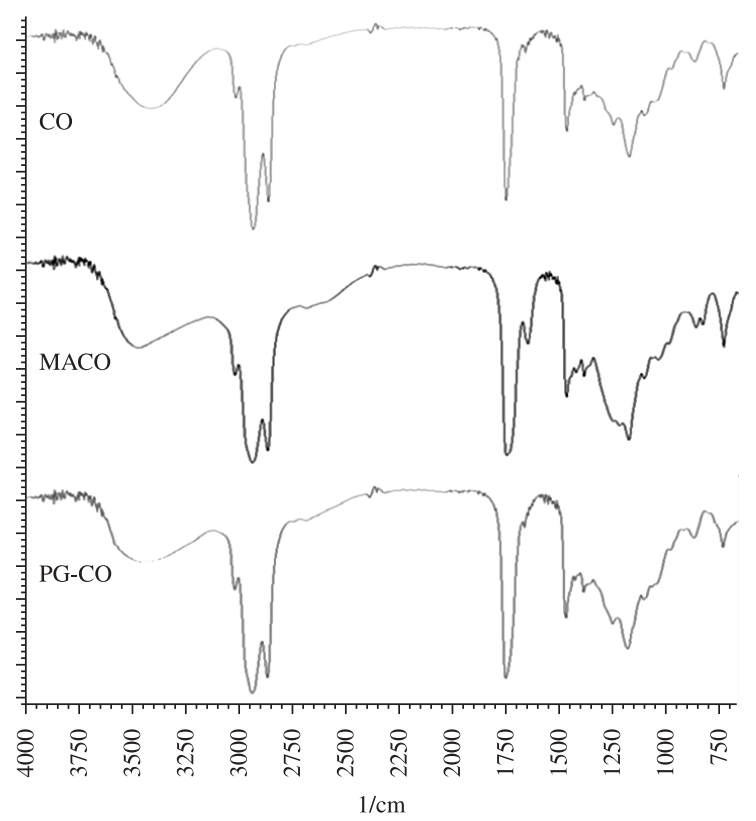

Figura 5. Espectros IR de CO (a), MACO con MW (b), PG-CO con MW (c).

\section{Influencia de las MW en la obtención del Aceite Transesterificado}

Debido a la alta polaridad del poliglicerol, es inmiscible con los aceites $\mathrm{CO}$ y MACO, por lo cual en las formulaciones de PU se requeriría una alta agitación y posiblemente calentamiento para miscibilizar las fases, esto sumado a la alta viscosidad del poliglicerol (54100 cps), hace que el proceso sea poco viable y las propiedades de las espumas finales pueden ser afectadas.
Para esto se realiza una transesterificación entre los dos, utilizando catalizadores básicos, en este trabajo se utiliza el mismo $\mathrm{NaOH}$ que se empleo en la síntesis del PG, la evidencia de que esta reacción se ha llevado a cabo es la formación de una sola fase, la cual se evalúa por inspección visual, durante el transcurso de la reacción.

Las microondas disminuyen el tiempo de reacción requerido para formar una sola fase, como se muestra en la Tabla 3, solo se requiere 2 horas, mientras que con calentamiento CONV se necesitan 6 horas. La disminución del valor de hidroxilos al final de la reacción, indica que ocurre pérdida por condensación de agua, los menores valores de $\mathrm{OHv}$ se obtienen con MW.

Los espectros IR obtenidos para el aceite de castor (CO), aceite de castor maleinizado (MACO) y el aceite transesterificado (PG-CO) (Figura 5), muestra que las señales características son: $3410 \mathrm{~cm}^{-1}$ estiramiento $\mathrm{OH}, 3012 \mathrm{~cm}^{-1}$ estiramiento $=\mathrm{CH}-, 2939$ y $2890 \mathrm{~cm}^{-1}$ estiramientos - $\mathrm{CH}-$ alcano, $1747 \mathrm{~cm}^{-1}$ estiramiento - $\mathrm{CO}-$ del triglicérido, 1454 y $1376 \mathrm{~cm}^{-1}$ flexiones simétricas de los grupos $\mathrm{CH}_{2}$ y $\mathrm{CH}_{3}$ respectivamente, 1242,1165 y $1100 \mathrm{~cm}^{-1}$ corresponden con estiramientos de -C-O-. En MACO aparece una señal a $1642 \mathrm{~cm}^{-1}$ de grupos $-\mathrm{C}=\mathrm{C}-$ por la incorporación del anhídrido maléico.

\section{Síntesis y caracterización de las espumas rígidas}

Los valores obtenidos para los experimentos y sus propiedades se describen en la Tabla 4, puede observarse que: la resistencia a la compresión presenta mayores valores en las espumas que tienen PG-MACO, esto es debido a que MACO tiene dentro de su estructura ácidos que pueden reaccionar con el diisocianato, generando grupos amida los cuales aumentan la resistencia mecánica.

Los valores de densidad son ligeramente menores en las espumas con PG-MACO, esto es debido a que los ácidos libres pueden formar $\mathrm{CO}_{2}$ adicional. La influencia del agua y el ácido tartárico será descrita más adelante.

Se utilizó el estadístico $t$ para cada una de las propiedades. Los $p$-valores mayores que 0.05 indican que no hay relaciones estadísticamente significativas entre las variables a un nivel de confianza del $95 \%$. Los valores obtenidos para los coeficientes con su respectivo p-valor se reportan en la Tabla 5.

Los términos que no son significativos de acuerdo a la prueba $t$ se resaltan en la Tabla 6, para todas las superficies el termino xy no es significativo. También se aprecia en el análisis de varianza que el modelo de regresión es significativo, y de acuerdo a los coeficientes de determinación $\mathrm{R}^{2}$ y Raj $^{2}$ el modelo explica bien la variabilidad presente en los datos. También se aprecia el error estándar de estimación (S) que dada la escala de medición de las variables de respuesta, tienen una magnitud relativamente pequeña.

Los puntos estacionarios obtenidos para la densidad son silla y sus valores se escapan del margen permitido para la síntesis de las espumas, porque un nivel de agua tan alto produce espumas de baja resistencia mecánica y no tiene entrecruzamiento. Los puntos obtenidos para la resistencia a la compresión son máximos y permiten obtener espumas de buena calidad, estos datos se reportan en la Tabla 6. 
Tabla 5. Coeficientes obtenidos para las superficies de respuesta.

\begin{tabular}{|c|c|c|c|c|c|c|c|c|}
\hline & $\begin{array}{c}\text { Constante } \\
\text { p-valor }\end{array}$ & $\begin{array}{c}\mathbf{X} \\
\text { p-valor }\end{array}$ & $\begin{array}{c}\mathbf{Y} \\
\text { p-valor }\end{array}$ & $\begin{array}{c}x^{2} \\
\text { p-valor }\end{array}$ & $\begin{array}{c}\mathbf{y}^{2} \\
\text { p-valor }\end{array}$ & $\begin{array}{c}\text { xy } \\
\text { p-valor }\end{array}$ & $\begin{array}{c}\mathbf{R}^{2} \\
\mathbf{R a j}^{2} \\
\mathbf{S} \\
\end{array}$ & $\begin{array}{c}\text { Análisis varianza } \\
\text { p-valor }\end{array}$ \\
\hline \multirow{3}{*}{$\begin{array}{c}\text { D } \\
\text { PG-CO }\end{array}$} & 70.456 & -18.967 & 4.883 & 1.026 & -1.624 & \multirow{2}{*}{-0.225} & 93.07 & \multirow{3}{*}{0.000} \\
\hline & \multirow{2}{*}{0.000} & \multirow{2}{*}{0.000} & \multirow{2}{*}{0.033} & \multirow{2}{*}{0.719} & \multirow{2}{*}{0.572} & & 88.74 & \\
\hline & & & & & & 0.925 & 4.635 & \\
\hline \multirow{3}{*}{$\begin{array}{c}\text { D } \\
\text { PG-MACO }\end{array}$} & \multirow{3}{*}{$\begin{array}{c}67.932 \\
0.000\end{array}$} & \multirow{3}{*}{$\begin{array}{c}-19.200 \\
0.000\end{array}$} & 5.567 & 1.121 & -1.379 & 0.300 & 95.52 & \multirow{3}{*}{0.000} \\
\hline & & & 0.007 & 0.629 & 0.554 & 0.877 & 92.71 & \\
\hline & & & & & & & 3.756 & \\
\hline $\mathrm{RC}$ & 242.029 & -21.467 & 51.267 & -18.818 & -25.918 & -7.875 & 95.34 & \multirow{3}{*}{0.000} \\
\hline \multirow{2}{*}{ PG-CO } & \multirow{2}{*}{0.000} & \multirow{2}{*}{0.002} & \multirow{2}{*}{0.000} & \multirow{2}{*}{0.030} & \multirow{2}{*}{0.007} & \multirow{2}{*}{0.227} & 92.43 & \\
\hline & & & & & & & 12.033 & \\
\hline \multirow{3}{*}{$\begin{array}{c}\text { RC } \\
\text { PG-MACO }\end{array}$} & 249.088 & -20.283 & 52.767 & -16603 & -26.153 & -5875 & 95.66 & \\
\hline & 0.000 & 0.003 & 0.000 & 0.043 & 0.005 & 0.342 & 92.94 & 0.000 \\
\hline & & & & & & 0.342 & 11.631 & \\
\hline
\end{tabular}

Tabla 6. Puntos estacionarios obtenidos de la optimización de la superficie de respuesta.

\begin{tabular}{lccccc}
\hline & $\begin{array}{c}\mathbf{x i} \\
\text { codificado }\end{array}$ & $\begin{array}{c}\text { yi } \\
\text { codificado }\end{array}$ & $\begin{array}{c}\mathbf{X i} \\
\text { real }\end{array}$ & $\begin{array}{c}\text { Yi } \\
\text { real }\end{array}$ & $\begin{array}{c}\text { Tipo de } \\
\text { punto }\end{array}$ \\
\hline D & 9.3325 & 0.8572 & 11.3325 & 3.2501 & Silla \\
PG-CO & & & & & \\
D & 8.1777 & 2.9071 & 10.1777 & 6.8374 & Silla \\
PG-MACO & & & & & \\
RC & -0.8028 & 1.1100 & 1.1972 & 3.6925 & Máximo \\
PG-CO & & & & & \\
$\begin{array}{l}\text { RC } \\
\text { PG-MACO }\end{array}$ & -0.8053 & 1.0993 & 1.1947 & 3.6738 & Máximo \\
\hline
\end{tabular}

Cuantificando las propiedades de las espumas empleando los modelos obtenidos (Tabla 5 y Figura 6), podemos ver que: la densidad disminuye al aumentar el contenido de agua por la formación de una mayor cantidad de $\mathrm{CO}_{2}$ que expande la espuma, pero no se ve muy influenciada por la cantidad de ácido tartárico, el cual al neutralizarse con la base que hay en el poliol, forma una sal que actúa como catalizador favoreciendo la formación de poliisocianuratos, los cuales hacen parte de la red polimérica, este favorece el entrecruzamiento y no la espumación, además los hidroxilos secundarios presentes en este reaccionan con el isocianato aumentando la densidad de entrecruzamiento. El agua reacciona formando grupos urea, cuando su concentración es muy alta se separan de la red formando microdominios, que hacen que el polímero pierda propiedades mecánicas y aumente la friabilidad.

De acuerdo con la optimización realizada se seleccionaron los niveles de agua: 3 pph y cantidad de tartárico de $1.75 \mathrm{pph}$, para obtener dos espumas rígidas cuya caracterización se describe en la Tabla 7. Puede verse que la densidad es mayor que las espumas convencionales petroquímicas, esto se debe a que no se empleó agentes halocarbonados para realizar la espumación. La resistencia a la compresión es mayor debido al alto entrecruzamiento, la conductividad térmica es más alta porque las espumas obtenidas presentan mayor densidad que las espumas comerciales y el $\mathrm{CO}_{2}$ generado, que es el responsable de aislar la temperatura, presenta una mayor conductividad térmica que los agentes espumantes usados en la industria.

La Figura 7 muestra las micrografías electrónicas de las espumas rígidas, donde puede verse que estas tienen celdas parcialmente abiertas, con pared celular y una arquitectura basada en aristas. La espuma convencional presenta el tamaño de celda más pequeño
Propiedades PU para tártarico $=0 \mathrm{pph}$

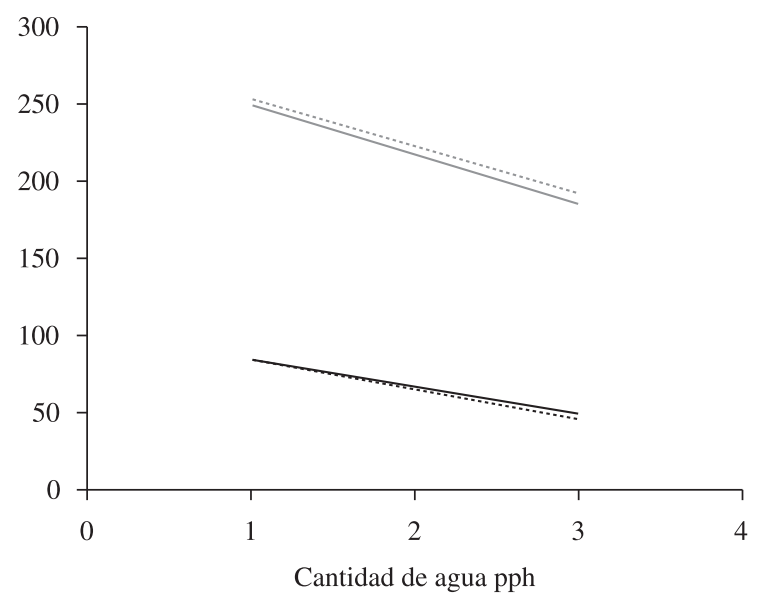

Propiedades PU para agua $=3 \mathrm{pph}$

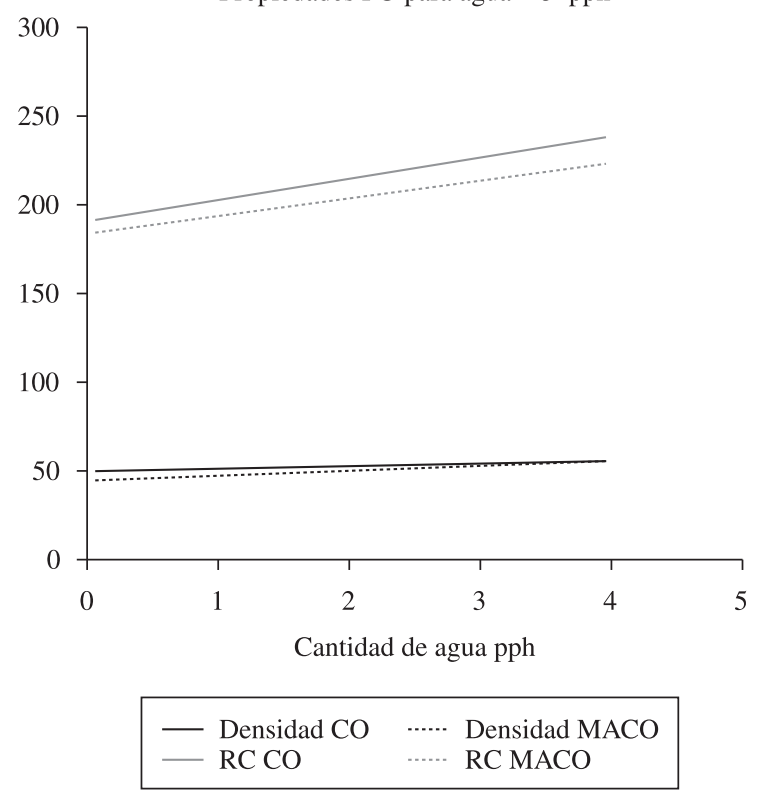

Figura 6. Cuantificación de las propiedades de las espumas rígidas, empleando las superficies de respuesta. 
Tabla 7. Caracterización espumas rígidas óptimas.

\begin{tabular}{ccccc}
\hline & $\begin{array}{c}\text { Densidad } \\
\left(\mathbf{K g . m}^{-3}\right)\end{array}$ & $\begin{array}{c}\text { RC } \\
(\mathbf{K P a})\end{array}$ & $\begin{array}{c}\text { Factor K } \\
\left(\mathbf{W . m K}^{-1}\right)\end{array}$ & \%Friabilidad \\
\hline PG-CO & 50.21 & 202.75 & 0.045 & 20.5 \\
PG-MACO & 45.78 & 212.34 & 0.045 & 17.4 \\
$\begin{array}{c}\text { Espuma rígida } \\
\text { convencional }\end{array}$ & 31 & 205.9 & 0.023 & 5 \\
\hline
\end{tabular}

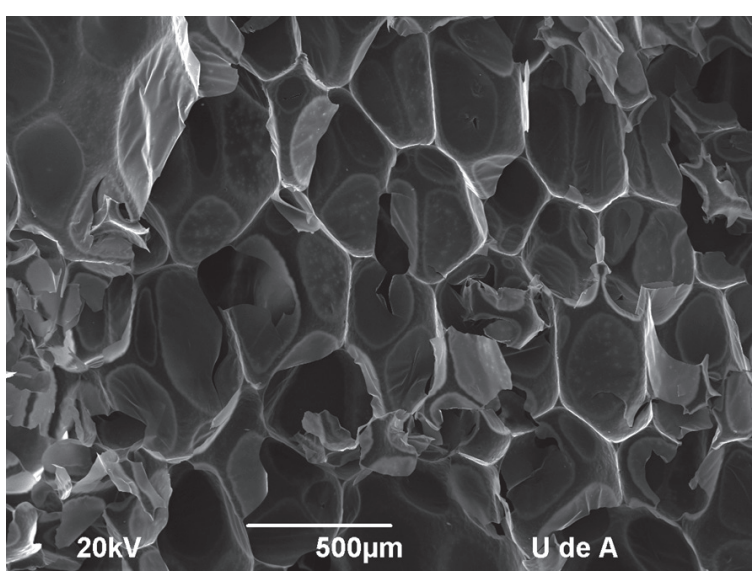

PG-MACO

(a)

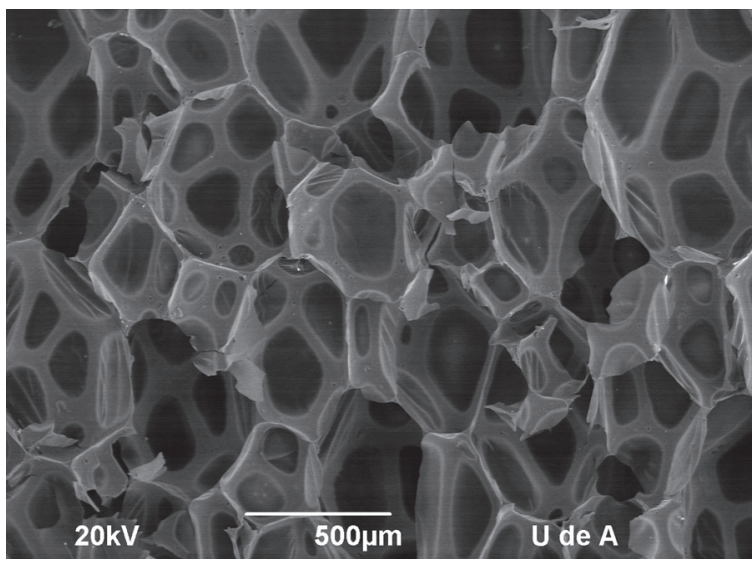

PG-CO

(b)

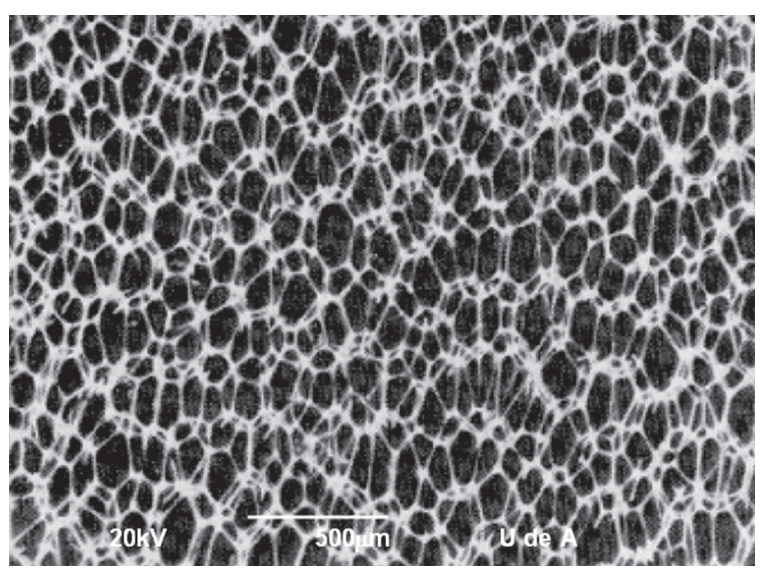

Espuma rígida convencional

(c)

Figura 7. Micrografías por SEM de las espumas rígidas obtenidas.

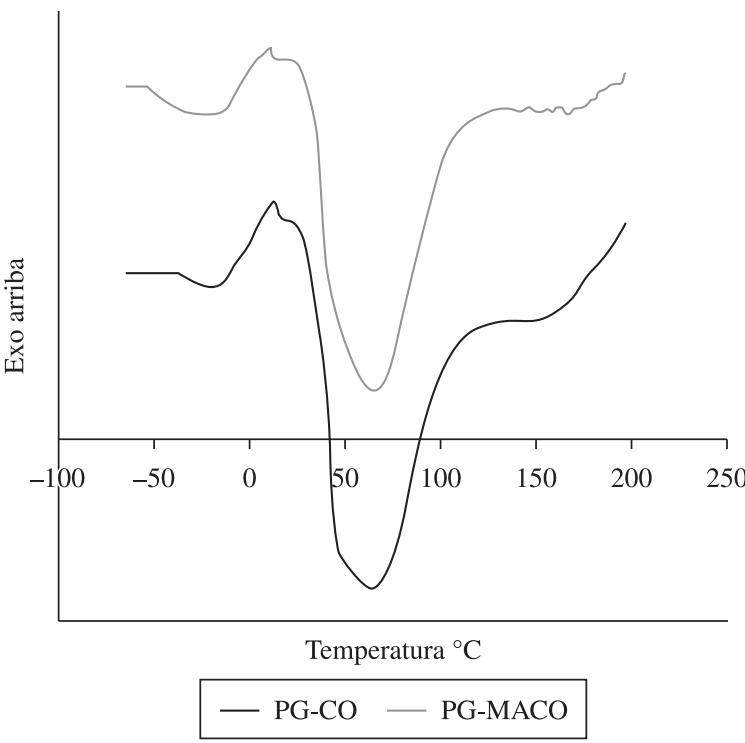

Figura 8. Análisis DSC para las espumas rígidas.

$(150 \mu \mathrm{m})$, la espumas de PG-CO $(245 \mu \mathrm{m})$ y PG-MACO $(250 \mu \mathrm{m})$, tienen valores similares.

El análisis de calorimetría diferencial de barrido de las espumas, muestra que estas presentan una temperatura de transición vítrea y un pico endotérmico en valores muy similares, para la espuma con poliol PG-CO el valor es de $\mathrm{Tg}=9.42{ }^{\circ} \mathrm{C}$ y $\mathrm{Tf}=56.56{ }^{\circ} \mathrm{C}$, y para PG-MACO la $\mathrm{Tg}=8.14{ }^{\circ} \mathrm{C}$ y $\mathrm{Tf}=59.42{ }^{\circ} \mathrm{C}$. Estos picos endotérmicos se deben a procesos de fusión de los segmentos cristalinos generados por la agregación molecular de alta energía cohesiva e interacciones intermoleculares como puentes de hidrogeno de los segmentos rígidos del polímero (uretano) con los oxígenos del poliglicerol. La formación de urea dentro de la red también forma agregados con altas fuerzas intermoleculares, que pueden fundir en un amplio rango de temperaturas. Los DSC se muestran en la Figura 8.

\section{Conclusiones}

Las microondas como fuente de calentamiento se presentan como una buena alternativa para acelerar las reacciones que tienen reactivos con alta constante dieléctrica o con mecanismos polares de intermedios iónicos. En este trabajo no se favorece la formación de aceite maleinizado de castor, pero si la formación de poliglicerol y la transesterificación de los aceites de CO y MACO con PG.

Las espumas rígidas obtenidas presentan mejor resistencia a la compresión que las espumas convencionales, pero presentan menor conductividad térmica debido a su estructura de celda abierta, por lo cual se recomienda su uso como aislante acústico. Son espumas con alta friabilidad, esta propiedad puede mejorarse disminuyendo la cantidad de ácido tartárico en las formulaciones.

Las espumas tienen un carácter termoplástico y presentan muchos grupos fácilmente hidrolizables lo que puede favorecer la biodegradabilidad, el reciclaje químico y mecánico de las mismas.

\section{Referencias}

1. Woods, G. W. - “The ICI polyurethanes book”, John-Wiley, Netherlands (1987).

2. Carme, M.; Babb D. \& Ryan, A. - Polymer., 49, p.3279 (2008).

3. Oertel, G. - "Polyurethane handbook", 2nd ed, Hanser Publishers, Munich (1994). 
4. Randall, D. \& Lee, S. - "The polyurethane book", John-Wiley, The United Kingdom (2002).

5. Vilar, W. - "Química y tecnología de poliuretanos", 3. ed., Río de Janeiro (2002).

6. Szycher, M. - "Szycher's handbook of polyurethanes", CRC Press, Boca Ratón- Florida, USA (1999).

7. Mazo, P.; Franco, A.; Rios, L. \& Restrepo, G. - Scientia et Technica., 13, p.607 (2007)

8. Mazo, P.; Estenoz, D \& Rios, L. - Polímeros, 19, p.149 (2009).

9. Mazo, P.; López, L.; Restrepo, D. \& Rios, L. - Polímeros, 20, p.134 (2010).

10. Wurm, F.; Nieberle, J. \& Frey, H. - Macromolecules., 41, p.1909 (2008).

11. Wilms, D.; Stiriba, S. \& Frey, H. - Accounts of chemical research., 43, p.129 (2010).

12. Kainthan, R. K.; Muliawan, E. B.; Hatzikiriakos, S. G. \& Brooks, D. E. - Macromolecules., 39, p.7708 (2006).
13. Jena, K. K.; Raju, K. V. S. N.; Prathab, B. \& Aminabhavi, T. M. - J. Phys. Chem. B., 111, p.8801 (2007).

14. Vigen, B. "Process for preparation and purification of polyglycerols and esters thereof". US Patent 3637774 (1972).

15. Da Silva, G. P.; Mack, M. \& Contiero, J. - Biotechnol. Adv., 27,p.30 (2009).

16. Zoran, P.; Mihail, I \& Ivan, J. - "Polyglycerol based polyols and polyurethanes and methods for producing polyols and polyurethanes", US Patent 2009/0082483 A1 (2009).

17. Kappe, O. - Angew Chem. Int. Ed., 43, p.6250 (2004).

18. Lidström, P.; Tierney, J.; Wathey, B. \& Westman, J. - Tetrahedron., 57, p.9225 (2001).

19. Mazo, P. \& Rios, L. - Ing. Quim.., 33 (2008).

20. Mazo, P. \& Rios, L. - LAAR., 40, p.337 (2010).

21. Mazo, P. \& Rios, L. - LAAR., 40, p.343 (2010).

Enviado: $19 / 02 / 10$

Reenviado: $28 / 06 / 10$

Aceito: $31 / 07 / 10$

DOI: $10.1590 / \mathrm{S} 0104-14282011005000006$ 